\title{
When less is not more
}

\section{Critics of experimentation in nonhuman primates have used a variety of arguments to defend their views. Yet some of those arguments can be used to advocate the use of these animals in biomedical research.}

$\mathbf{T}$ he investigational use of nonhuman primates has long been a contentious issue. On the one hand, supporters of their use are genuinely convinced that insights derived from the study of monkeys and apes could never be obtained in other species, and there is a vast literature, particularly in disciplines like systems neuroscience, that supports such a claim. On the other hand, critics call for a blanket prohibition of experimentation on nonhuman primates on the basis of ethical concerns and of a litany of cases in which observations made in other primates have failed to correspond to the reality of human biology.

Regrettably, the debate has often turned sour, and cases of intimidation of and violence towards researchers who work on nonhuman primates are well known even to people with a casual interest in this matter. Just as regrettably, policymakers and other stakeholders have too often taken a relatively conservative stance on this problem, trying to please both advocates and critics by supporting the noncommittal idea that, although experimentation in nonhuman primates is necessary for the time being, it is desirable to pursue the development of alternatives to their use.

Are there realistic alternatives to the use of nonhuman primates in biomedical research? The development of in vitro and in silico approaches to replace monkeys and apes in the lab ranks very highly among the priorities of many critics. Unfortunately, this idea ignores at least two issues. First, as powerful as in vitro and computational strategies might be, they cannot shed as much light on the complex interactions between cells and organs as the use of whole animals can.

Second, most of the nonhuman primates currently studied in biomedical research are used for toxicological screening during the drug development process. For example, in 2005, 77\% of the nonhuman primates used in the UK were part of toxicological studies that evaluated the safety of potential new drugs. And, although there are many in vitro tests for prescreening a drug's potential toxicity, it's hard to see how in vitro approaches by themselves can supplant the use of nonhuman primates in the evaluation of drug safety.

Some would argue that in vitro models can ultimately deliver surrogate markers to predict in vivo safety. This may be so, but let's not forget that the discovery of surrogate markers of efficacy that could serve as alternative trial endpoints is the holy grail of clinical trials in humans. Alas, these markers are extraordinarily hard to come by, and it's not clear that surrogate markers of safety, as opposed to efficacy, would be any easier to uncover. If anything, they are probably harder to identify, particularly in the case of offtarget, non-mechanism-related side effects.

Others would argue that species such as dogs, ferrets and pigs could be used in toxicological studies instead of monkeys. These species are indeed employed, and increasing their use to replace nonhuman primates is certainly a possibility that needs to be explored. It is important to remember, though, that regulatory agencies often insist on the need for safety data from at least two species when considering new drugs. Which two species will satisfy the regulators and how much companies simply choose to play it safe by using monkeys as a matter of course are important issues that will inform this debate and that, so far, have received relatively little attention.

Related to the suggestion of using animal models different from nonhuman primates is the claim that research in monkeys and apes has been counterproductive for our understanding of human biology. As results from nonhuman primates do not always correspond to what is later observed in people, some critics have brandished this lack of correspondence as an argument that invalidates the use of nonhuman primates in research.

However, this argument ignores cases in which studies in nonhuman primates have indeed resulted in successful human therapies. In the case of Parkinson's disease, for example, the development of deep-brain stimulation - what many scientists in the field regard as the current therapeutic gold standardfollowed from experiments in a nonhuman primate model of the disease showing that destruction or high-frequency stimulation of the subthalamic nucleus reversed parkinsonian symptoms.

One additional problem with the 'lack of correspondence' criticism is that modeling human biology and pathology is a trial-and-error process. We don't know a priori if a given model, regardless of the species, will faithfully reproduce human biology or predict therapeutic efficacy in people. 


\section{EDITORIAL}

It's true that we can occasionally discount from the start the use of nonhuman primates as model systems, particularly in cases in which existing models in other species have already been successful in the translation of a basic discovery into a therapeutic advance. But for most conditions, and certainly for diseases for which there are no treatments, the process of finding the right model to develop a cure is one of the toughest challenges during the drug discovery process. To say that the failure of a model in one instance is a reason to abandon the model entirely ignores the magnitude of that challenge. Instead, a failure should be construed as a learning experience; if a clinical trial didn't work, one must try to understand the reason behind the failure and go back to the lab to improve upon the experiment. This is a key part of what translational research is about.

This is not to say that nonhuman primates must be regarded as the first port of call when trying to model human disease. As mentioned above, dogs, pigs, ferrets and other species have an important part to play in our efforts to understand human biology. However, the phylogenetic proximity between nonhuman primates and humans remains an inescapable fact that makes monkeys and apes particularly attractive as experimental models and raises the burden of proof necessary to rule out their potential usefulness.

In connection with this evolutionary closeness, critics have argued that such proximity makes it unethical to use nonhuman primates in research. It would be impossible to do justice to the ethics of the relationship between humans and animals—a topic that has been the focus of a lot of rigorous studies-in this brief discussion, but it is important to mention a couple of key points.

Several ethical arguments regarding the use of nonhuman primates revolve around their close resemblance to humans, closer than that of any other species. This line of reasoning, however, has problems. For example, several of the arguments involve concepts such as personhood, self-awareness and sociability, the definition of which in relation to nonhuman primates remains under debate by scholars who work on this topic. It would therefore be premature to draw definitive conclusions about our ethical responsibility to nonhuman primates on the basis of our current understanding of the problem.

The ethical argument of genetic relatedness also has problems. Chimpanzees share $98.5 \%$ of their genes with humans, and the two species diverged from each other and from gorillas about 5 million years ago. Old World monkeys like the macaque-the monkey species most commonly experimented upon-diverged from the great apes about 20 million years ago. New World primates like the marmoset — another widely used species—-diverged from Old World primates about 35 million years ago. So, how far back in time shall we set the boundary of what should be regarded as an acceptable species to use? Any answer will ultimately be arbitrary. Furthermore, if we worry about our closest relatives, it should be noted that experiments in apes are very rare, and some countries, such as the UK, already set extraordinarily high bars to grant approval for their use, effectively banning them from research.

The problems of establishing the ethical responsibility of humans toward other primates do not negate the seriousness of this ethical dilemma. But it is important to bring them up in the context of another ethical problem that is easier to grasp: our responsibility to alleviate the suffering caused by the many diseases that affect humans. If research in nonhuman primates has the potential to benefit people, as suggested by supporters of the use of monkeys and apes and as evidenced by success stories like deep-brain stimulation, then our hard-to-establish ethical responsibility toward other primates cannot trump our unequivocal responsibility towards other human beings. And if we consider that the number of nonhuman primates used in research projects is always a very small fraction of the number of people affected by the disease under investigation, it shouldn't be difficult to decide on which side of the dilemma we must err.

We can go one step further and argue that, until the ethical discussion is solved, the resemblance argument can actually be used to promote the use of nonhuman primates in disciplines where it hasn't had much impact yet. For example, the study of psychiatric disorders has begun to focus on their fractionation into endophenotypes-simpler disease 'units' more amenable to experimental approaches. It is not difficult to imagine that we will be able to model some of these endophenotypes only in monkeys and apes precisely because of their phylogenetic proximity to humans. Although the prospect of studying in nonhuman primates what could be construed as quintessentially human features may be disturbing for some, the solid scientific case that can be made to support the use of monkeys and apes in research must take precedence over ethical arguments until the latter can be settled for good. 